\title{
REVIEW
}

\section{Simulation in neonatal care: towards a change in traditional training?}

\author{
Nadya Yousef ${ }^{1}\left[\right.$ [Domain Moreau $^{1} \cdot$ Lamia Soghier $^{2,3}$
}

Received: 16 November 2021 / Revised: 30 December 2021 / Accepted: 2 January 2022 / Published online: 12 January 2022

(c) The Author(s), under exclusive licence to Springer-Verlag GmbH Germany, part of Springer Nature 2022

\begin{abstract}
Simulation has traditionally been used in neonatal medicine for educational purposes which include training of novice learners, maintaining competency of health care providers, and training of multidisciplinary teams to handle crisis situations such as neonatal resuscitation. Current guidelines recommend the use of simulation as an education tool in neonatal practice. The place of simulation-based education has gradually expanded, including in limited resource settings, and is starting to show its impact on improving patient outcomes on a global basis. Over the past years, simulation has become a cornerstone in clinical settings with the goal of establishing high quality, safe, reliable systems. The aim of this review is to describe neonatal simulation training as an effective tool to improve quality of care and patient outcomes, and to encourage the use of simulation-based training in the neonatal intensive care unit (NICU) for not only education, but equally for team building, risk management and quality improvement.

Conclusion: Simulation is a promising tool to improve patient safety, team performance, and ultimately patient outcomes, but scarcity of data on clinically relevant outcomes makes it difficult to estimate its real impact. The integration of simulation into the clinical reality with a goal of establishing high quality, safe, reliable, and robust systems to improve patient safety and patient outcomes in neonatology must be a priority.

\section{What is Known:}

- Simulation-based education has traditionally focused on procedural and technical skills.

- Simulation-based training is effective in teaching non-technical skills such as communication, leadership, and teamwork, and is recommended in neonatal resuscitation.

What is New:

- There is emerging evidence for the impact of simulation-based training on patient outcomes in neonatal care, but data on clinically relevant outcomes are scarce.

- Simulation is a promising tool for establishing high quality, safe, reliable, and robust systems to improve patient safety and patient outcomes.
\end{abstract}

Keywords Simulation · Neonatal care · Resuscitation · Quality improvement · Teamwork · Education · Newborn

\section{Communicated by Daniele De Luca}

Nadya Yousef

nadya.yousef@aphp.fr

Romain Moreau

romain.moreau.abc@gmail.com

Lamia Soghier

lsoghier@childrensnational.org

1 Division of Pediatrics and Neonatal Critical Care, Dept of Perinatal Medicine, "A.Béclère" Medical Center, Paris Saclay University Hospitals, APHP, Paris, France

2 Department of Pediatrics, The George Washington University School of Medicine and Health Sciences, Washington, DC, USA

3 Department of Neonatology, Children's National, Washington, DC, USA

\author{
Abbreviations \\ NRP Neonatal resuscitation program \\ NICU Neonatal intensive care unit \\ ELBW Extra low birth weight infants \\ HBB Helping Babies Breathe program \\ QI Quality improvement \\ NBP Neonatal brachial plexus \\ MRSA Methicillin-resistant Staphylococcus aureus \\ ECMO Extracorporeal membrane oxygenation
}

\section{Background}

Simulation has a long history and tradition in medical education. In eighteenth century France, using the world's first simulator, Madame du Coudray trained over 10,000 midwifery students with the objective of reducing infant 
mortality rates [1]. The use of simulation-based training in medical education has since steadily progressed, particularly in high-risk specialties such as anesthesia, obstetrics, and neonatal-perinatal medicine primarily in high-income countries mainly due to the cost of the simulation equipment [2]. Although simulation-based training has lagged in limited resource settings [3], large-scale global programs have shown its success [4]. Additionally, simulation-based training plays a critical role in patient safety. "To Err is Human" [5] underlined the importance of raising the level of patient safety with a shift from a traditional view of focusing on individual practitioner weaknesses to a systems approach focusing on the underlying system failures to prevent patient harm. Human factors and non-technical skills such as leadership, communication, and teamwork are therefore critical factors in improving team performance and in preventing medical errors [6]. Consequently, simulation has evolved over the recent years to become an essential tool in the training of teams, and is increasingly being used in quality improvement (QI) initiatives and to enhance safety culture [2]. According to Dr. Halamlek, "Our mission in neonatology — the delivery of safe, effective, and efficient care to critically ill neonates-can be greatly enhanced through the thoughtful and systematic application of simulation-based training, assessment, and research" [7].

The aim of this review is to describe the use of neonatal simulation training as a tool in improving provider skills and team performance, and to discuss its potential in improving quality of care, patient safety, and patient outcomes in the neonatal intensive care unit (NICU).

\section{Simulation-based training and education}

Simulation-based training offers a safe risk-free environment for clinicians to develop and maintain competency [8]. Simulation-based training has become an integral part of many neonatal-perinatal medicine training programs, and is used as a basis for many standardized newborn training programs worldwide to teach neonatal procedures and resuscitation as noted in the 2021 European Resuscitation Council Guidelines [9-11]. In addition, simulation has the potential to play a key role in maintaining professional competency for infrequently performed high-risk procedures such as thoracentesis and extracorporeal membrane oxygenation (ECMO) $[12,13]$.Simulation-based training is also a valuable tool in teaching behavioral skills such as effective communication, teamwork, and leadership, which are essential aspects of team performance. Simulation can be used to develop these non-technical skills that cannot be learned using traditional teaching methods, and can be used to improve confidence and knowledge [8, 14].

Simulation in training and education can take many forms. It can be provided in dedicated simulation laboratories/centers or on-site in the health care setting, hospital, or clinic [15]. Simulation can be scheduled as specifically designated training sessions, or built-into the practice of a functioning unit, e.g., mock codes during working hours [16]. The use of newer technology such as virtual reality, tele-simulation, and serious games is increasing and may play a useful part in training of health care providers particularly for distance learning [17, 18]. Many examples have emerged during the COVID-19 pandemic attesting to the versatility of simulation even when performed remotely [19].

Fidelity is the level of realism associated with a particular simulation activity [20]. Fidelity is important for learner engagement and can be achieved through the use of appropriate material (mannequins, task trainers, software), by recreating an environment that resembles the clinical setting, through well-designed scenarios with which learners can relate (semantic fidelity) and which trigger cognitive and psychological responses [21, 22, 8, 18, 23]. In-situ simulation is conducted in the actual clinical environment using clinical equipment and resources that closely mirror the clinical reality $[13,22,24]$. It can also be used to identify latent threats to patient safety and to test new spaces [25-27].

When designing simulation-based education sessions and scenarios, a teaching plan which includes the learning objectives is essential. Learning objectives are not only central to scenario design but assist in the choice of the task trainer or mannequin to use [2, 18, 23]. In addition, the decision on whether to use high or low technology material is influenced by the clinical setting and local resources. There are a variety of neonatal task trainers for practicing, e.g., bag and mask ventilation, endotracheal intubation, and catheter placement. There is no conclusive data showing the superiority of high vs low-technology mannequins for simulation training in neonatology [8]. Each has its specific uses and should align with the desired learning outcomes. Despite the increase in the number of commercially available mannequins and task trainers, there is an unmet need for advanced neonatal simulators that allow for clinicians to practice neonatal scenarios. It is however possible to use simple and inexpensive materials to customize the mannequins to simulate specific neonatal conditions such as gastroschisis and meningomyeloceles $[18,23,28]$.

Post-simulation debriefing is one of the most important components of simulation-based education and is critical to the learning experience [29]. Its objective is to encourage the learners' reflective thinking and provide feedback about performance. Debriefing provides learners the possibility to reformulate the experienced scenario, explain thought processes, discuss and learn from mistakes, and identify unmet needs $[29,30,30-32]$. The science of debriefing has rapidly 
evolved over the past decades. Facilitator-guided debriefing is the most common method used in debriefing and the one that has been most extensively studied [30, 33]. Debriefing methods encourage learners to discuss salient aspects of their simulated performance with minimal guidance from facilitators who encourage reflective learning. The facilitator's role is primarily to ensure that learners realize all the scenario learning objectives and through their own reflection internalize triumphs and failures. Several debriefing models such as advocacy inquiry, PEARLS, and plus-delta (outcomes-based), amongst others, have been described $[30,34,35]$. All focus on deep self-driven learner reflection on action as described by Kolb [36]. There is currently no evidence for the best method for debriefing, although determining ways to debrief based on specific learning contexts and specific learner needs seems to be a good approach to conduct effective debriefings [30].

\section{Simulation and procedural skills training}

Procedures remain a cornerstone of neonatal care. In the delivery room, approximately $1 \%$ of neonates will need advanced resuscitation interventions, with up to $15 \%$ of ELBW needing cardiopulmonary resuscitation [37, 38]. Most preterm infants and sick term babies will need additional procedures during their hospital stay. Adequate competency in neonatal procedures is critical to the quality of neonatal care. Personnel should be trained for procedures occurring on a regular basis (e.g., central line placement) and on low incidence emergency procedures (e.g., chest tube insertion, thoracentesis, pericardiocentesis, and arrhythmias) to which teams are infrequently exposed. In these cases, training not only allows development of procedural skills for trainees and inexperienced personnel, but also is essential for the ongoing training of experienced personnel to maintain professional competency. In recent years, optimal management practices of newborn infants have gradually shifted towards a more individualized approach and a decrease in the number of invasive procedures. In addition, restrictions in working hours have resulted in decreased exposure to emergency situations for trainees.

Procedural skills training can occur as a stand-alone training, or in large training sessions encompassing many disciplines and many different stations, in the form of boot camps [23, 39]. However, results obtained by simulationbased assessment methods of practical skills may be difficult to translate into clinical practice, even when using validated assessment tools [40]. Additional challenges relate to retention of knowledge and skill, and underline the importance of repeated training to maintain proficiency [41-43].

Simulation is also used to train teams in the cognitive, technical, and behavioral skills needed for complex low-frequency, high-risk activities such as ECMO where it has been shown to effectively improve clinical performance [44-46]. Di Nardo et al. studied team performance in an Italian pediatric intensive care unit after initiation of high technology simulation training for ECMO. The study showed a decrease in oxygenator change time after failure from 5.3 to $3.9 \mathrm{~min}(P=0.02)$, a decrease in the time to respond to an air embolism $(P=0.048)$, and an increase in behavioral scores after simulation-based training $(P<0.01)$ [46]. These results confirm a previous study by $\mathrm{Su}$ et al. that shows improvement in the deployment time of extracorporeal cardiopulmonary resuscitation from 51 to $40 \mathrm{~min}(P=0.018)$ after the use of simulation-based training and the application of formal quality improvement tools to the process [47]. However, both studies are of low sample size which emphasizes the need for further research into patient outcomes following simulation-based training.

\section{Simulation and team training in neonatal resuscitation}

Neonatal resuscitation is an example of a high acuity, high occurrence situation. Multidisciplinary resuscitation teams need to be trained and prepared to ensure optimal performance in, often unanticipated, high-stress situations with high stakes [8].

Even in busy academic centers, it is challenging to ensure adequate training of all staff and maintain a consistent high level of skills and preparedness. Strict adherence to resuscitation guidelines is difficult [48-50].

Simulation-based education has emerged as an excellent tool to train teams in resuscitation compared to traditional clinical training [8]. Simulation is now an integral part of many standardized neonatal training programs worldwide [11]. The Neonatal Resuscitation Program (NRP) was the first resuscitation program to integrate simulation-based training into its curriculum. Using a standardized curriculum including debriefing, the program has since been introduced to vast body of providers in the USA, and been made accessible to many disciplines across the globe through traditional NRP® training and the Helping Babies Breath (HBB) curriculum and through development of its supporting mannequins [18] The current European Resuscitation Council guidelines now recommend the use of simulation in training programs $[11,51]$.

In a neonatal resuscitation setting, teams are usually multidisciplinary, with professionals from different backgrounds often coming together to work for the first time. Team behavior and team communications significantly influence the quality of neonatal resuscitation, and have been implicated in the majority of medical errors, with negative consequences on perinatal morbidity and mortality $[52,53]$. 
Simulation-based training can be used to train non-technical skills such as communication, teamwork, and leadership to improve team performance and team coordination [8].

There are few studies evaluating team performance after simulation training in neonatal resuscitation [53, 54]. A recent review found significantly improved team performance in simulated re-evaluation 3 and 6 months after simulation training [53].

\section{Simulation and patient outcomes}

Medical simulation strives to improve patient outcomes. However, data directly focusing on direct patient outcomes are few, especially in neonatal care $[53,55]$. In addition, outcomes such as improvement of non-practical skills and human factors are difficult to evaluate and may be underreported $[55,56]$. Examples from the adult and pediatric literature show that simulation-based education has led to improvement in certain patient outcomes such as a decrease in "time to needle" in stroke patients [57], a decrease in time to blood transfusion in trauma patients [58], improved clinical response to postpartum hemorrhage [59], and increased survival to discharge after cardiac arrest [60].

Available reports on the effect of simulation on direct patient outcomes in neonatal care are often limited to single center studies. Several reviews on the impact of simulation on neonatal mortality and morbidity have not been able to reveal effects of team training on neonatal outcomes because of insufficient or poor quality data [53,61].

The following examples highlight the use of simulationbased training to improve neonatal outcomes:

\section{Simulation, central line-associated bloodstream infection (CLABSI), and hospital acquired infections}

Central line-associated bloodstream infections (CLABSIs) are common problems in the NICU and are associated with increased mortality rates. Studies from 2012 have shown that CLABSI rates can decrease significantly from 13.9 per 1000 catheter days to $4.7(P<0.0001)$ in a NICU after integrating simulation-based training into a CLABSI prevention bundle [62]. This data is confirmed by adult studies showing CLABSI rates declining by $85 \%$ in a medical intensive care unit after implementation of a simulation-based training protocol [63]. In this instance, the same simulation-based curriculum was successfully disseminated and implemented in a community hospital with a significant improvement in CLABSI rates [64]. Interestingly, CLABSI rates rose again after a short time period when residents were untrained and dropped again after the training program was reinstituted. This indicates that simulation-based training is highly effective in reduction of hospital acquired infections.
In situ simulation has also been shown to mitigate a methicillin-resistant $S$. aureus outbreak in a newly renovated NICU [65]. In this study, all NICU providers underwent a brief in-situ training session that included education, evaluation, and debriefing. The study reported improved hand hygiene compliance and improvement in the culture of safety during a 6-month period. The in situ simulation intervention helped identify latent safety risks and improved workflow. No new cases of MRSA colonization or infection occurred over the course of 3 weeks after the simulation.

\section{Simulation and reduction of hypothermia in extremely low birth weight infants in the first hour after admission to the NICU}

The care of the extremely low birth infant (ELBW) is challenging and requires a high level of skill and teamwork. A French monocentric, retrospective, mixed-methods study evaluated the impact of an interdisciplinary in-situ simulation focusing on the "Golden Hour" (the first hour after admission to the NICU), on hypothermia and the time to surfactant administration after birth, which are both associated with outcomes in the ELBW $[37,66]$. A hundred and thirty team members underwent interprofessional in situ simulation targeting the management of the ELBW infant during the first hour of life (Golden Hour protocol) over a period of 17 months (Fig. 1). Results after training showed improvement in body temperature $\left(36.4{ }^{\circ} \mathrm{C}\right.$ [35.7-36.9] versus $35^{\circ} \mathrm{C}$ [34-36]; $\left.P<0.0001\right)$ and a decrease in time to surfactant administration $(2.5 \mathrm{~h} \mathrm{[2.5-3]}$ versus $3.5 \mathrm{~h}$ [3-4]; $P=0.001$ ) after the training [67]. The study also included a qualitative analysis of the related learning processes and behavioral changes observed in relation to the simulation sessions. Improvement in team work and communication were observed after team training [68].

\section{Simulation and prevention of neonatal brachial plexus injury}

Birth trauma is a low-frequency high severity event. Multidisciplinary training by obstetrical providers using simulation training for management of shoulder dystocia has been shown to decrease the incidence of neonatal brachial plexus (NBP) injury. A meta-analysis of 10 studies (nonrandomized and 1 cluster randomized trial) showed that the percentage of neonatal brachial plexus injuries per shoulder dystocia case fell from 12.1 to $5.7 \%$ (RR, $0.37 ; 95 \% \mathrm{Cr}$, $0.26-0.57$; probability of reduction $100 \%$ ) in 3121 shoulder dystocia deliveries [69]. NBP injuries were also reduced across all deliveries (both vaginal and $\mathrm{C}$ section) but to a smaller extent (RR, 0.53 ; $95 \% \mathrm{Cr}, 0.21-1.26$; probability of 
Fig. 1 (C) 2021 Premasim, Romain Moreau. All rights reserved. With permission. Photos from Premasim, an in situ simulation program at the APHP Paris Saclay University NICU in Paris. A A low technology mannequin (Premature Anne ${ }^{\mathrm{TM}}$ ) and clinical equipment from the NICU are used to create a realistic experience for learners. B Ongoing scenario with a multiprofessional team. C Low cost "icebreakers." Plastic cups with sweets covered with various slogans are used to facilitate briefing and debriefing. D Debriefing
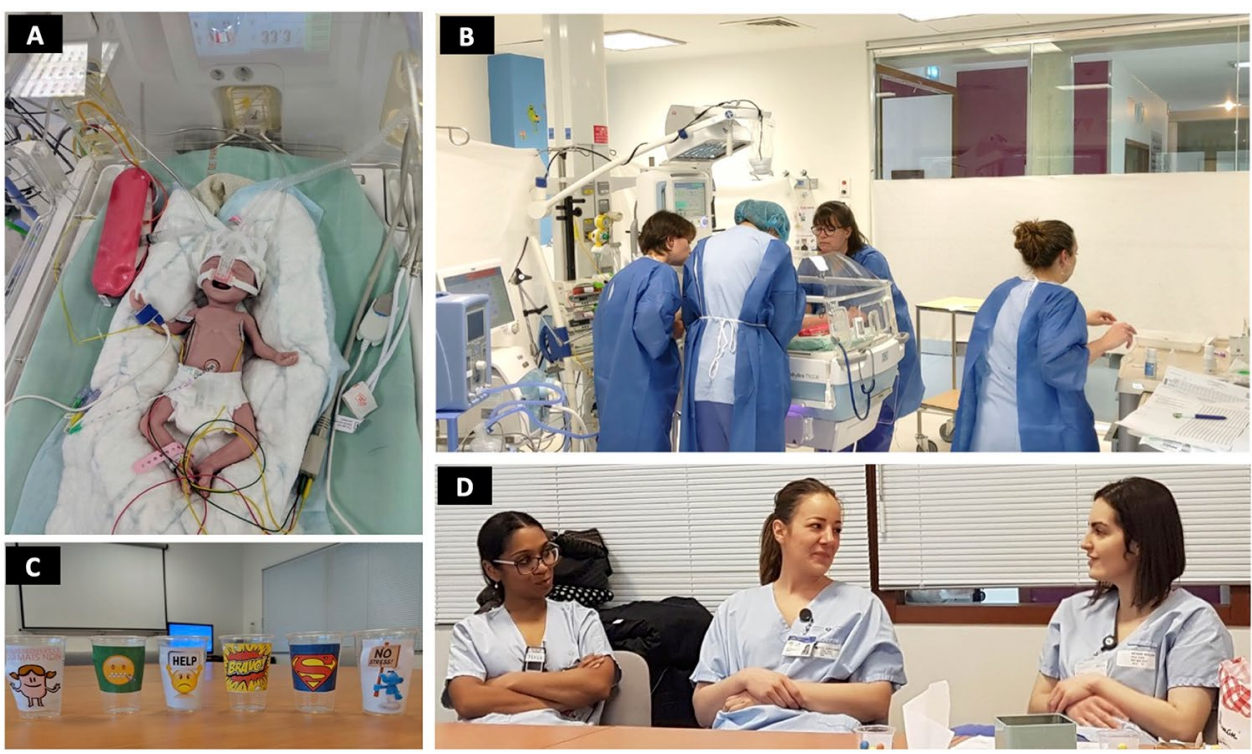

reduction 94\%) [69]. Clavicular and humeral fractures were reduced in 6 studies (RR, 0.56 ; $95 \% \mathrm{Cr}, 0.27-1.18$; probability of reduction $94 \%$ ) of 2275 cases of shoulder dystocia after simulation training. There was a marked variability in simulation exercises, type of mannequin, and frequency of sessions making it difficult to standardize the type of training that would be needed to see similar results, and the costs of simulation training were not calculated. The effect of simulation training was most noticeable immediately after introduction of these programs, but the effect on patient outcomes declined thereafter [70].

\section{Simulation and reduction in neonatal mortality and stillbirth rates}

Use of simulation training for obstetric and neonatal management of preterm births (and infants $>1000 \mathrm{~g}$ birthweight) in a cluster randomized trial (PRONTO training) has been shown to decrease neonatal mortality and stillbirth rates in Uganda and Kenya (control (23.3\%) vs intervention (15.3\%) OR 0.66 (CI 0.54-0.81) $<0.0001$ [71]. This difference remained even after adjustment for caesarean sections, multiple gestations, sex, country, birthweight, center delivery volume, and facility readiness. The training which occurred every 5-8 weeks included scenarios addressing gestational age assessment, kangaroo care, breastfeeding, neonatal resuscitation, and feeding for preterm infants.

Helping Babies Breathe (HBB) is an example of a simulation program using low fidelity equipment to teach neonatal resuscitation in limited resource settings [72]. The program trains birth attendants in the essential skills of neonatal resuscitation and has been implemented globally. HBB has been shown to improve early neonatal outcomes and fresh stillbirths in several settings, but its impact is difficult to measure because of lack of reliable data [61]. HBB has been shown to be highly cost-effective in a study from a Tanzanian rural hospital [73]. This again highlights the gains that could be achieved by simulation training even in limited resource environments.

\section{Challenges in neonatal simulation}

Interdisciplinary simulation training is an ideal tool for improving the quality of neonatal care. Several barriers still remain and should be addressed in order to fully integrate simulation into the wider clinical setting. Common barriers to implementation include cost, time, organizational challenges, unwillingness of staff, and absence of support from policy makers [22]. There are still no clear studies showing cost effectiveness of simulation [74]. Cost of material may be attenuated through innovative solutions to adapt available material to the desired learning objectives, or to use available equipment from the clinical setting which can be recycled for repeated use, or by sharing simulation material and equipment with other teams. Development of sharing programs or rental programs could be an innovative solution to the financial problem. Organizational challenges such as poor staffing and/or limited availability of time are a reality in many settings and must be addressed on an institutional level [22]. The justification of the use of simulation in training and in quality improvement initiatives is hampered by the current scarcity of evidence on clinically relevant outcomes and the absence of research on cost-effectiveness. Research into these areas is crucial to facilitate dialogue with decision-makers and stakeholders, and to urge integration of simulation into the areas of training, risk management and QI. 


\section{Conclusion}

Simulation has evolved from a procedural training tool for novices to slowly becoming a cornerstone in clinical settings in spite of numerous challenges. Simulation is a promising tool to improve patient safety, team performance, and ultimately patient outcomes, but scarcity of data on clinically relevant outcomes makes it difficult to estimate its real impact. The integration of simulation into the clinical reality with a goal of establishing high quality, safe, reliable, and robust systems to improve patient safety and patient outcomes must be a priority.

Research on cost effectiveness is essential to achieve buyin from decision makers and stakeholders.

Although many challenges and many unanswered questions still remain, the future seems promising. As per Dr. Gaba "The future of simulation in health care depends on the commitment and ingenuity of the health care simulation community to see that improved patient safety using this tool becomes a reality" [2].

Acknowledgements Authors are grateful to Dr. Shivani ShankarAguilera and Dr. Emmanuelle Letamendia for their constructive comments on the manuscript.

Authors' contributions N. Yousef and L. Soghier conceived and designed the review, performed the literature search, and wrote the first manuscript draft. R. Moreau helped with the literature search, critically reviewed the manuscript, and prepared the figures. All authors reviewed the manuscript for important intellectual content, agreed to be held accountable for all aspects of the work, and approved the final manuscript version to be submitted.

\section{Availability of data and material N/A.}

Code availability N/A.

\section{Declarations}

\section{Ethical approval N/A.}

Consent to participate N/A.

\section{Consent for publication N/A.}

Conflict of interest N. Yousef has served as a lecturer and received grants and honoraria from Chiesi Pharmaceuticals, and has served as a member of advisory boards for Chiesi Pharmaceuticals and for Radiometer Medical ApS. These companies have had no role in the design, preparation, review, or approval of the manuscript or the decision to submit it for publication. R. Moreau has received honoraria from Emergensim. The company has had no role in the design, preparation, review, or approval of the manuscript or the decision to submit it for publication. L. Soghier is the editor of the Neonatal Simulation and receives royalties from the American Academy of Pediatrics for this work. Dr. Soghier is also grant funded from the National Institute of Health on projects related to development of computer augmented neonatal intubation simulators.

\section{References}

1. Carty E (2010) Educating midwives with the world's first simulator: Madame du Coudray's Eighteenth Century Mannequin 9:11

2. Gaba DM (2004) The future vision of simulation in health care. Qual Saf Health Care 13:i2-i10. https://doi.org/10.1136/qshc. 2004.009878

3. Abass Olalere A, Samuel Babafemi O, Odufeko Gabriel T (2014) Medical simulation a tool yet untapped in most developing nations in Africa. Int J Comput Appl 97:1-4. https://doi.org/10.5120/ 17000-7136

4. Dol J, Campbell-Yeo M, Murphy GT et al (2018) The impact of the Helping Babies Survive program on neonatal outcomes and health provider skills: a systematic review. JBI Database Syst Rev Implement Rep 16:701-737. https://doi.org/10.11124/JBISRIR-2017-003535

5. America I of M (US) C on Q of HC in, Kohn LT, Corrigan JM, Donaldson MS (2000) To err is human. National Academies Press (US)

6. Charney C (2011) Making a team of experts into an expert team. Adv Neonatal Care Off J Natl Assoc Neonatal Nurses 11:334-339. https://doi.org/10.1097/ANC.0b013e318229b4e8

7. Halamek LP (2016) Simulation and debriefing in neonatology 2016: mission incomplete. Semin Perinatol 40:489-493. https:// doi.org/10.1053/j.semperi.2016.08.010

8. Garvey AA, Dempsey EM (2020) Simulation in neonatal resuscitation Front Pediatr 8:59. https://doi.org/10.3389/fped.2020.00059

9. Sawyer T, Stavroudis TA, Ades A et al (2020) Simulation in neonatal-perinatal medicine fellowship programs. Am J Perinatol 37:1258-1263. https://doi.org/10.1055/s-0039-1693465

10. Dempsey E, Pammi M, Ryan AC, Barrington KJ (2015) Standardised formal resuscitation training programmes for reducing mortality and morbidity in newborn infants. Cochrane Database Syst Rev CD009106. https://doi.org/10.1002/14651858.CD009106.pub2

11. Greif R, Lockey A, Breckwoldt J et al (2021) European Resuscitation Council Guidelines 2021: education for resuscitation. Resuscitation 161:388-407. https://doi.org/10.1016/j.resuscitation.2021. 02.016

12. Barsuk JH, Cohen ER, Williams MV et al (2018) Simulationbased mastery learning for thoracentesis skills improves patient outcomes: a randomized trial. Acad Med J Assoc Am Med Coll 93:729-735. https://doi.org/10.1097/ACM.0000000000001965

13. Johnston L, Oldenburg G (2016) Simulation for neonatal extracorporeal membrane oxygenation teams. Semin Perinatol 40:421429. https://doi.org/10.1053/j.semperi.2016.08.002

14. Dempsey E, Pammi M, Ryan AC, Barrington KJ (2015) Standardised formal resuscitation training programmes for reducing mortality and morbidity in newborn infants. Cochrane Database Syst Rev. https://doi.org/10.1002/14651858.CD009106.pub2

15. Sørensen JL, Østergaard D, LeBlanc V et al (2017) Design of simulation-based medical education and advantages and disadvantages of in situ simulation versus off-site simulation. BMC Med Educ 17:20. https://doi.org/10.1186/s12909-016-0838-3

16. Blakely TG (2007) Implementing newborn mock codes. MCN Am J Matern Child Nurs 32:230-235; quiz 236-237. https://doi.org/ 10.1097/01.NMC.0000281962.56207.44

17. Ghoman SK, Patel SD, Cutumisu M et al (2020) Serious games, a game changer in teaching neonatal resuscitation? A review. Arch Dis Child - Fetal Neonatal Ed 105:98-107. https://doi.org/10. 1136/archdischild-2019-317011

18. Soghier L, Robin B (2021) Neonatal simulation: a practical guidelPaperback, 1st edn. American Academy of Pediatrics, USA

19. Mileder LP, Bereiter M, Wegscheider T (2021) Telesimulation as a modality for neonatal resuscitation training. Med Educ Online 26:1892017. https://doi.org/10.1080/10872981.2021.1892017 
20. Lioce L (2020) Healthcare Simulation Dictionary, Second. Agency for Healthcare Research and Quality

21. Dieckmann P, Gaba D, Rall M (2007) Deepening the theoretical foundations of patient simulation as social practice. Simul Healthc J Soc Simul Healthc 2:183-193. https://doi.org/10.1097/ SIH.0b013e3180f637f5

22. Kurup V, Matei V, Ray J (2017) Role of in-situ simulation for training in healthcare: opportunities and challenges. Curr Opin Anaesthesiol 30:755-760. https://doi.org/10.1097/ACO.0000000000000514

23. Johnston LC, Campbell D, Manhas D (2016) Simulation for neonatal care. In: Grant VJ, Cheng A (eds) Comprehensive healthcare simulation: pediatrics. Springer International Publishing, Cham, pp 231-244

24. Goldshtein D, Krensky C, Doshi S, Perelman VS (2020) In situ simulation and its effects on patient outcomes: a systematic review. BMJ Simul Technol Enhanc Learn 6:3-9. https://doi.org/ 10.1136/bmjstel-2018-000387

25. Bender GJ (2011) In situ simulation for systems testing in newly constructed perinatal facilities. Semin Perinatol 35:80-83. https:// doi.org/10.1053/j.semperi.2011.01.007

26. Kjaergaard-Andersen G, Ibsgaard P, Paltved C, Irene Jensen H (2021) An in situ simulation program: a quantitative and qualitative prospective study identifying latent safety threats and examining participant experiences. Int J Qual Health Care J Int Soc Qual Health Care 33:mzaa148. https://doi.org/10.1093/intqhe/mzaa148

27. Auerbach M, Stone KP, Patterson MD (2016) The role of simulation in improving patient safety. In: Grant VJ, Cheng A (eds) Comprehensive Healthcare Simulation: Pediatrics. Springer International Publishing, Cham, pp 55-65

28. Bruno CJ, Glass KM (2016) Cost-effective and low-technology options for simulation and training in neonatology. Semin Perinatol 40:473-479. https://doi.org/10.1053/j.semperi.2016.08.008

29. Fanning RM, Gaba DM (2007) The role of debriefing in simulation-based learning. Simul Healthc J Soc Simul Healthc 2:115125. https://doi.org/10.1097/SIH.0b013e3180315539

30. Sawyer T, Eppich W, Brett-Fleegler M et al (2016) More than one way to debrief: a critical review of healthcare simulation debriefing methods. Simul Healthc J Soc Simul Healthc 11:209-217. https://doi.org/10.1097/SIH.0000000000000148

31. Grant VJ, Cheng A (2016) Comprehensive healthcare simulation: pediatrics. Springer International Publishing, Cham

32. Bauchat J, Seropian M (2020) Essentials of debriefing in simulation-based education. In: Comprehensive health care simulation: anesthesiology. Springer Nature, Switzerland

33. Gardner R (2013) Introduction to debriefing. Semin Perinatol 37:166-174. https://doi.org/10.1053/j.semperi.2013.02.008

34. Eppich W, Cheng A (2015) Promoting Excellence and Reflective Learning in Simulation (PEARLS): development and rationale for a blended approach to health care simulation debriefing. Simul Healthc J Soc Simul Healthc 10:106-115. https://doi.org/10.1097/ SIH.0000000000000072

35. Rudolph JW, Simon R, Rivard P et al (2007) Debriefing with good judgment: combining rigorous feedback with genuine inquiry. Anesthesiol Clin 25:361-376. https://doi.org/10.1016/j.anclin. 2007.03.007

36. Kolb DA (1984) Experiential learning: experience as the source of learning and development. Prentice-Hall, Englewood Cliffs, NJ

37. Wyckoff MH, Aziz K, Escobedo MB et al (2015) Part 13: Neonatal resuscitation. Circulation 132:S543-S560. https://doi.org/10. 1161/CIR.0000000000000267

38. Wyckoff MH, Salhab WA, Heyne RJ et al (2012) Outcome of extremely low birth weight infants who received delivery room cardiopulmonary resuscitation. J Pediatr 160:239-244.e2. https:// doi.org/10.1016/j.jpeds.2011.07.041

39. Wayne DB, Cohen ER, Singer BD et al (2014) Progress toward improving medical school graduates' skills via a "boot camp" curriculum. Simul Healthc J Soc Simul Healthc 9:33-39. https:// doi.org/10.1097/SIH.0000000000000001

40. Sawyer T, Gray MM (2016) Procedural training and assessment of competency utilizing simulation. Semin Perinatol 40:438-446. https://doi.org/10.1053/j.semperi.2016.08.004

41. Matterson HH, Szyld D, Green BR et al (2018) Neonatal resuscitation experience curves: simulation based mastery learning booster sessions and skill decay patterns among pediatric residents. J Perinat Med 46:934-941. https://doi.org/10.1515/jpm-2017-0330

42. Yeung J, Djarv T, Hsieh MJ et al (2020) Spaced learning versus massed learning in resuscitation - a systematic review. Resuscitation 156:61-71. https://doi.org/10.1016/j.resuscitation.2020. 08.132

43. Donoghue A, Navarro K, Diederich E et al (2021) Deliberate practice and mastery learning in resuscitation education: a scoping review. Resusc Plus 6:100137. https://doi.org/10.1016/j.resplu. 2021.100137

44. Raffaeli G, Ghirardello S, Vanzati M et al (2018) Start a neonatal extracorporeal membrane oxygenation program: a multistep team training. Front Pediatr 6:151. https://doi.org/10.3389/fped.2018. 00151

45. Johnston LC, Su L (2021) Comprehensive healthcare simulation: ECMO simulation: a theoretical and practical guide. Springer International Publishing, Cham

46. Di Nardo M, David P, Stoppa F et al (2018) The introduction of a high-fidelity simulation program for training pediatric critical care personnel reduces the times to manage extracorporeal membrane oxygenation emergencies and improves teamwork. J Thorac Dis 10:3409-3417. https://doi.org/10.21037/jtd.2018.05.77

47. Su L, Spaeder MC, Jones MB et al (2014) Implementation of an extracorporeal cardiopulmonary resuscitation simulation program reduces extracorporeal cardiopulmonary resuscitation times in real patients. Pediatr Crit Care Med J Soc Crit Care Med World Fed Pediatr Intensive Crit Care Soc 15:856-860. https://doi.org/ 10.1097/PCC.0000000000000234

48. Mitchell A, Niday P, Boulton J et al (2002) A prospective clinical audit of neonatal resuscitation practices in Canada. Adv Neonatal Care Off J Natl Assoc Neonatal Nurses 2:316-326. https://doi.org/ 10.1053/adnc.2002.36831

49. Maya-Enero S, Botet-Mussons F, Figueras-Aloy J et al (2018) Adherence to the neonatal resuscitation algorithm for preterm infants in a tertiary hospital in Spain. BMC Pediatr 18:319. https:// doi.org/10.1186/s12887-018-1288-3

50. Schilleman K, Siew ML, Lopriore E et al (2012) Auditing resuscitation of preterm infants at birth by recording video and physiological parameters. Resuscitation 83:1135-1139. https://doi.org/ 10.1016/j.resuscitation.2012.01.036

51. Madar J, Roehr CC, Ainsworth S et al (2021) European Resuscitation Council Guidelines 2021: newborn resuscitation and support of transition of infants at birth. Resuscitation 161:291-326. https://doi.org/10.1016/j.resuscitation.2021.02.014

52. Sentinel Event Alert 30: Preventing infant death and injury during delivery I The Joint Commission. https://www.jointcommission. org/resources/patient-safety-topics/sentinel-event/sentinel-eventalert-newsletters/sentinel-event-alert-issue-30-preventing-infantdeath-and-injury-during-delivery/ Accessed 16 Nov 2021

53. Lindhard MS, Thim S, Laursen HS et al (2021) Simulation-based neonatal resuscitation team training: a systematic review. Pediatrics 147:e2020042010. https://doi.org/10.1542/peds.2020-042010

54. Rubio-Gurung S, Putet G, Touzet S et al (2014) In situ simulation training for neonatal resuscitation: an RCT. Pediatrics 134:e790797. https://doi.org/10.1542/peds.2013-3988

55. McGaghie WC, Draycott TJ, Dunn WF et al (2011) Evaluating the impact of simulation on translational patient outcomes. Simul Healthc J Soc Simul Healthc 6:S42-S47. https://doi.org/10.1097/ SIH.0b013e318222fde9 
56. Brazil V (2017) Translational simulation: not 'where?' but 'why?' A functional view of in situ simulation. Adv Simul 2:20. https:// doi.org/10.1186/s41077-017-0052-3

57. Ajmi SC, Advani R, Fjetland L et al (2019) Reducing door-toneedle times in stroke thrombolysis to 13 min through protocol revision and simulation training: a quality improvement project in a Norwegian stroke centre. BMJ Qual Saf 28:939-948. https:// doi.org/10.1136/bmjqs-2018-009117

58. Gray A, Chartier LB, Pavenski K et al (2021) The clock is ticking: using in situ simulation to improve time to blood administration for bleeding trauma patients. CJEM 23:54-62. https://doi.org/10. 1007/s43678-020-00011-9

59. de Melo BCP, Van der Vleuten CPM, Muijtjens AMM et al (2021) Effects of an in situ instructional design based postpartum hemorrhage simulation training on patient outcomes: an uncontrolled before-and-after study. J Matern-Fetal Neonatal Med Off J Eur Assoc Perinat Med Fed Asia Ocean Perinat Soc Int Soc Perinat Obstet 34:245-252. https://doi.org/10.1080/14767058.2019. 1606195

60. Young AK, Maniaci MJ, Simon LV et al (2020) Use of a simulationbased advanced resuscitation training curriculum: impact on cardiopulmonary resuscitation quality and patient outcomes. J Intensive Care Soc 21:57-63. https://doi.org/10.1177/1751143719838209

61. Morris SM, Fratt EM, Rodriguez J et al (2020) Implementation of the helping babies breathe training program: a systematic review. Pediatrics 146:e20193938. https://doi.org/10.1542/peds. 2019-3938

62. Steiner M, Langgartner M, Cardona F et al (2015) Significant reduction of catheter-associated blood stream infections in preterm neonates after implementation of a care bundle focusing on simulation training of central line insertion. Pediatr Infect Dis J 34:1193-1196. https://doi.org/10.1097/INF.0000000000000841

63. Barsuk JH, Cohen ER, Feinglass J et al (2009) Use of simulationbased education to reduce catheter-related bloodstream infections. Arch Intern Med 169:1420. https://doi.org/10.1001/archinternmed. 2009.215

64. Barsuk JH, Cohen ER, Potts S et al (2014) Dissemination of a simulation-based mastery learning intervention reduces central line-associated bloodstream infections. BMJ Qual Saf 23:749756. https://doi.org/10.1136/bmjqs-2013-002665

65. Gibbs K, DeMaria S, McKinsey S et al (2018) A novel in situ simulation intervention used to mitigate an outbreak of methicillin-resistant Staphylococcus aureus in a neonatal intensive care unit. J Pediatr 194:22-27.e5. https://doi.org/10.1016/j.jpeds.2017. 10.040
66. Bahadue FL, Soll R (2012) Early versus delayed selective surfactant treatment for neonatal respiratory distress syndrome. Cochrane Database Syst Rev 11:CD001456. https://doi.org/10. 1002/14651858.CD001456.pub2

67. Moreau R, Yousef N, Poteaux N, Deluca D (2021) Amélioration de la qualité des soins et gestion des risques par la simulation in situ en réanimation néonatale [Quality improvement and risk management using in situ simulation in neonatal intensive care units]. Proceedings from the JFRN Conference. Paris (abstract)

68. Romain Moreau (2021) Genèse et effets d'un dispositif de formation interprofessionnelle par simulation in situ sur la qualité de prise en charge du nouveau-né très grand prématuré lors de la golden hour en réanimation néonatale. Strasbourg

69. Wagner SM, Bell CS, Gupta M et al (2021) Interventions to decrease complications after shoulder dystocia: a systematic review and Bayesian meta-analysis. Am J Obstet Gynecol 225:484.e1-484.e33. https://doi.org/10.1016/j.ajog.2021.05.008

70. van de Ven J, Fransen AF, Schuit E et al (2017) Does the effect of one-day simulation team training in obstetric emergencies decline within one year? A post-hoc analysis of a multicentre cluster randomised controlled trial. Eur J Obstet Gynecol Reprod Biol 216:79-84. https://doi.org/10.1016/j.ejogrb.2017.07.020

71. Walker D, Otieno P, Butrick E et al (2020) Effect of a quality improvement package for intrapartum and immediate newborn care on fresh stillbirth and neonatal mortality among preterm and low-birthweight babies in Kenya and Uganda: a clusterrandomised facility-based trial. Lancet Glob Health 8:e1061e1070. https://doi.org/10.1016/S2214-109X(20)30232-1

72. Budhathoki SS, Gurung R, Ewald U et al (2019) Does the Helping Babies Breathe Programme impact on neonatal resuscitation care practices? Results from systematic review and meta-analysis. Acta Paediatr 108:806-813. https://doi.org/10.1111/apa.14706

73. Vossius C, Lotto E, Lyanga S et al (2014) Cost-effectiveness of the "helping babies breathe" program in a missionary hospital in rural Tanzania. PLoS ONE 9:e102080. https://doi.org/10.1371/ journal.pone.0102080

74. Zendejas B, Wang AT, Brydges R et al (2013) Cost: the missing outcome in simulation-based medical education research: a systematic review. Surgery 153:160-176. https://doi.org/10.1016/j. surg.2012.06.025

Publisher's Note Springer Nature remains neutral with regard to jurisdictional claims in published maps and institutional affiliations. 